\title{
LINES AND OSCULATING LINES OF HYPERSURFACES
}

\author{
J.M. LANDSBERG AND C. ROBLES
}

\begin{abstract}
This is a detailed study of the infinitesimal variation of the varieties of lines and osculating lines through a point of a low degree hypersurface in projective space. The motion is governed by a system of partial differential equations which we describe explicitly.
\end{abstract}

\section{INTRODUCTION}

1.1. Motivation and context. Let $X^{n} \subset \mathbb{C P}^{n+1}$ be a hypersurface with $\operatorname{deg}(X) \leq n$. Given a point $x \in X$, let $\mathcal{C}_{x} \subset \mathbb{P} T_{x} X$ denote the tangent directions to lines $\ell=\mathbb{P}^{1}$ such that $x \in \ell \subset X$. If $\operatorname{deg}(X) \leq 2$ and $x, y \in X$ are general points, then $\mathcal{C}_{x}$ is projectively isomorphic to $\mathcal{C}_{y}$. The goal of this paper is to answer a question posed by Jun-Muk Hwang:

Let $\operatorname{deg}(X) \geq 3$. How can $\mathcal{C}_{x}$ vary (modulo projective isomorphism) as $x$ varies over the general points of $X$ ?

The question is motivated by Hwang and N. Mok's program to study Fano varieties via the variety of tangent directions to minimal degree rational curves through a point. (See [Hwa01] for an overview.)

The first interesting case is $d=3$ and $n=4$. Here $\mathcal{C}_{x}$ is a curve of degree six in $\mathbb{P}^{3}$ - i.e., a genus four curve in its canonical embedding. When $d=3$ and $n=5, \mathcal{C}$ is a $K 3$ surface in $\mathbb{P}^{4}$.

In another direction, this paper is a continuation of a program to understand the relationship between the algebraic geometry of subvarieties of projective space and their local projective differential geometry. This was a project of the classical geometers (e.g. [Fub16, Sev01, Car92]), was revived by Griffiths and Harris in [GH79] and continued in [JM94, Lan99b, Lan03a, Lan99a, Lan99c, Rob08, LR08, AG05, AG04, FP01], for example.

Before addressing Hwang's question, it will be useful to generalize it as follows: Fix $d \leq n$ and let $X^{n} \subset \mathbb{P}^{n+1}$ be a variety of degree at least $d$. Given $x \in X$, let $\mathcal{C}_{d, x} \subset \mathbb{P} T_{x} X$ denote the tangent directions to lines osculating to order $d$ with $X$ at $x$. (See $\S 2$ for a precise definition and a discussion of osculation). If $d=\operatorname{deg}(X)$, then $\mathcal{C}_{d, x}=\mathcal{C}_{x}$. For sufficiently general $X$ of degree at least $d$ and general $x \in X, \mathcal{C}_{d, x} \subset \mathbb{P} T_{x} X$ is the transverse intersection of smooth hypersurfaces of degrees $2, \ldots, d$. In particular it has codimension $d-1$ in $\mathbb{P} T_{x} X$.

1.2. The results. Our results are technical and require substantial notation to state precisely. Here we present central ideas and roughly state the results. The terminology will be made precise in Sections 2 and 3.

Let $X \subset \mathbb{P}^{n+1}$ be a hypersurface of degree at least $d$. We say $x \in X$ is a $d$-general point if all discrete projective differential invariants up to order $d$ are constant on a Zariski

Date: October 7, 2008. 
open subset of $X$ containing $x$. Let $p$ denote the Hilbert polynomial of $\mathcal{C}_{d, x} \subset \mathbb{P} T_{x} X$. Let $U \subset X$ be a neighborhood of $x$ that admits a local framing (cf. $\S 2.2$ ) consisting of $d$-general points. A choice of a local framing $e$ yields, for all $y \in U$, polynomials $\left(F_{2, e, y}, \ldots, F_{d, e, y}\right)$, with $F_{\delta, e, y} \in S^{\delta} T_{y}^{*} X$. While the polynomials depend on our choice of frame, their zero set in $\mathbb{P} T_{y} X$ does not; that variety is $\mathcal{C}_{d, y} \subset \mathbb{P} T_{y} X$. The framing enables us to identify the tangent spaces at different points with a fixed complex vector space $T$ of dimension $n$. Then we may regard these polynomials as elements of $S^{\delta} T^{*}$. This determines a map

$$
\hat{\phi}_{d, e}: U \rightarrow \text { Hilb }_{p}
$$

where $\mathrm{Hilb}_{p}$ is the Hilbert scheme of subvarieties of $\mathbb{P} T=\mathbb{P}^{n-1}$ having Hilbert polynomial $p$.

In order to eliminate the ambiguity in our choice of framing (which determines the identification of $T_{x} X$ with $T$ ) we wish to quotient the image by the action of $G L(T)$. The quotient of $H_{i l b}$ by $G L(T)$ is not a manifold or algebraic variety. However, the quotient of $\hat{\phi}_{d, e}(U) \subset H i l b_{p}$ will usually be a manifold. For example, if $X$ is sufficiently general, the stabilizer in $G L(T)$ of a point in $\hat{\phi}_{d, e}(U)$ will be trivial. For simplicity of discussion, for the moment assume this is the case. Let $H_{i l b}^{0}$ denote the open subset of $H_{i l b}$ where the stabilizer in $G L(T)$ is trivial and such that $H i l b_{p}^{0}$ contains $\hat{\phi}_{d, e}(U)$ and is a manifold. Set

$$
\mathcal{M}_{d}^{p}=H_{i l b}^{0} / G L(T) .
$$

which by definition is a manifold. We obtain a well defined map

$$
\phi_{U, d}: U \rightarrow \mathcal{M}_{d}^{p} \text {. }
$$

This map is independent of choice of local framing, so it extends to a well-defined map

$$
\phi_{d}: X_{\text {general }} \rightarrow \mathcal{M}_{d}^{p} \text {. }
$$

For most $X$ the Hilbert polynomial $p$ will be that of a generic complete intersection of type $(2, \ldots, d)$ in $\mathbb{P}^{n-1}$, and the stabilizer $G$ will be trivial. (The Hilbert polynomial is that of a generic complete intersection if and only if the polynomials $F_{2, e, x}, \ldots, F_{d, e, x}$ have no non-trivial syzygies.) We denote this generic moduli space by $\mathcal{M}_{d}$. Describing $\mathrm{d} \phi_{d}$ in the case that $d$ is the degree of $X$ answers Hwang's question. We show

(Theorem 4.1.a) The image of $\phi_{d}$ satisfies a first-order system of partial differential equations. The system is expressed by the condition that the Gauss map $\gamma_{\phi_{d}}$ associated to $\phi_{d}$ takes image in a proper subvariety $\boldsymbol{\Xi}_{d}^{p}$ of the Grassmann bundle of $\mathcal{M}_{d}^{p}$. We give an explicit description of $\boldsymbol{\Xi}_{d}^{p}$ in $\S 4$.

(Theorem 4.1.b) When $\operatorname{deg}(X)=d$ (so that $\mathcal{C}_{x, d}=\mathcal{C}_{x}$ ) the image of $\phi_{d}$ satisfies a more restrictive first-order system of partial differential equations: the Gauss image must lie in a smaller subvariety $\boldsymbol{\Phi}_{d}^{p} \subset \boldsymbol{\Xi}_{d}^{p}$. We give an explicit description of $\boldsymbol{\Phi}_{d}^{p}$ in $\S 4$.

(Theorem 4.2) Suppose that $\operatorname{deg}(X) \geq d$ and that the Gauss image of $\phi_{d}\left(X_{\text {general }}\right)$ lies in $\boldsymbol{\Phi}_{d}^{p}$. Then, under mild genericity conditions on $X$, we may conclude that $\operatorname{deg}(X)=d$. (More precisely, it is sufficent to assume that either $X$ is smooth or that for a general line $\ell \subset X, \ell \cap X_{\text {sing }}=\emptyset$.)

(Theorem 4.5) There is a non-empty Zariski open subset $A_{d}$ of $\mathcal{M}_{d}$, such that for any $X$ with $\operatorname{deg}(X) \geq d \geq 4$ and $x \in X_{\text {general }}$ with $\phi(x) \in A_{d}, \operatorname{rank}\left(\left.\mathrm{d} \phi_{d}\right|_{x}\right)=n$. 
Theorem 4.5 is suprising as it gives conditions that imply that $\mathcal{C}_{x}$ "must move as much as possible" without any hypotheses on $X$.

In the special case that $d=3$, Theorem 4.1.b is related to a classical result of Fubini and Cartan. Fubini [Fub16] stated (and Cartan [Car20] gave a rigorous proof) that under the hypotheses of the theorem, $X$ is projectively determined by $\phi_{3}(U), U \subset X$ any open subset in the analytic topology. For a short, modern proof, see [JM94].

Remark 1.1. In [JM94] they also extend the Cartan-Fubini theorem when $n \geq 3$ to the case that the second fundamental form is degenerate. The result fails in the case of surfaces, see [Car20], though it still holds "generically."

The results above are consequences of Theorems 3.2 and 3.3 on the Fubini forms (cf. $§ 2.3$ ) of the hypersurface $X$. More precisely the $\mathcal{C}_{d, x}$ do not contain all the geometric information of $X$ accessible by $d$ derivatives at a point. This information is contained in the Fubini

forms $\left(F_{2, e, x}, \ldots, F_{d, e, x}\right)-\operatorname{cf} . \S 3$, with $F_{\delta, e, x} \in S^{\delta} T^{*}$. (The exception is the case $d=3$, where $\mathcal{C}_{x}$ contains all the information of the Fubini forms.) The collection of Fubini forms are not well-defined at $x \in X$, but do define an equivalence class under the action of a group $H$ of dimension $n^{2}+2 n+3$, see $\S 4.1$.

We will first establish results in a fixed local framing $e$ which fixes a choice of Fubini forms. In particular, we have a map

$$
\tilde{\phi}_{d, e}: U \rightarrow \oplus_{\delta=2}^{d} S^{\delta} T^{*} .
$$

We determine a first-order system of partial differential equations that $\tilde{\phi}_{d, e}$ must satisfy (Theorem 3.2). We quotient to the Hilbert scheme and then by the action of $G L(T)$ to obtain the choice-free results listed above. This is carried out in Section 4.

Several examples of cubic hypersurfaces are considered in $\S 5$.

\section{LOCAL FRAMES AND FUBINI FORMS}

2.1. Notational conventions. For subsets $X \subset \mathbb{P} V, \widehat{X} \subset V$ denotes the corresponding cone. For a submanifold $X \subset \mathbb{P} V$ and $x \in X, \widehat{T}_{x} X \subset V$ denotes its affine tangent space. The tangent space and normal space at $x$ are

$$
T_{x} X=\hat{x}^{*} \otimes\left(\widehat{T}_{x} X / \hat{x}\right) \quad \text { and } \quad N_{x} X=T_{x} \mathbb{P} V / T_{x} X=\hat{x}^{*} \otimes\left(V / \widehat{T}_{x} X\right),
$$

respectively.

We will use the following index ranges:

$$
\begin{aligned}
& 0 \leq j, k, \ell \leq n+1, \\
& 1 \leq a, b, a_{j} \leq n, \\
& \mathrm{~N}=n+1 .
\end{aligned}
$$

The linear span of vectors $\left\{v_{1}, \ldots, v_{k}\right\}$ is denoted $\left\langle v_{1}, \ldots, v_{k}\right\rangle$. 
2.2. Adapted frames. Let $\pi: \mathbb{C}^{n+2} \backslash 0 \rightarrow \mathbb{P}^{n+1}$ denote the natural projection $v \mapsto[v]$. Let $X^{n} \subset \mathbb{P}^{n+1}$ be a submanifold and let $\mathcal{F}^{1} \rightarrow X$ denote the bundle of first-order adapted frames. Elements of $\mathcal{F}^{1} \subset \mathrm{GL}(V)$ are frames (or bases) $e=\left(e_{0}, e_{1}, \ldots, e_{\mathrm{N}}\right)$ of $V=\mathbb{C}^{n+2}$, such that

$$
\begin{aligned}
\pi\left(e_{0}\right) & \in X, \\
\widehat{T}_{\left[e_{0}\right]} X & =\left\langle e_{0}, \ldots, e_{n}\right\rangle .
\end{aligned}
$$

A local, first-order adapted framing is a section $e: U \rightarrow \mathcal{F}^{1}, U \subset X$ open in the analytic topology. Every smooth point on a projective variety admits a neighborhood $U$ with local framing ( $(2.4)$.

Since $X$ is a hypersurface, $N_{x} X$ is a line bundle spanned by

$$
\underline{e}_{\mathrm{N}}:=e^{0} \otimes\left(e_{\mathrm{N}} \bmod \widehat{T}_{e_{0}} X\right) \text {. }
$$

Here $\left(e^{0}, \ldots, e^{\mathrm{N}}\right)$ is the basis of $V^{*}$ dual to $e$.

Define the $\mathfrak{g l}(V)$-valued Maurer-Cartan form $\omega=\omega_{k}^{j} e_{j} \otimes e^{k}$ on $G L(V)$ by $\mathrm{d} e_{j}=\omega_{j}^{k} e_{k}$. Recall the Maurer-Cartan equation:

$$
\mathrm{d} \omega_{k}^{j}=-\omega_{\ell}^{j} \wedge \omega_{k}^{\ell} .
$$

We abuse notation and denote the pullback of $\omega$ to $\mathcal{F}^{1}$ by $\omega$ as well.

2.3. Fubini forms. The variety $\mathcal{C}_{k, x}$ will be defined (Definition 2.2) as the zero set of the Fubini forms $\left(F_{2, e, x}, \ldots, F_{k, e, x}\right)$. We review Fubini forms here; see [IL03, Ch. 3] for details.

Since $\widehat{T}_{\left[e_{0}\right]} X=\left\langle e_{0}, e_{1}, \ldots, e_{n}\right\rangle$ we have $\omega_{0}^{\mathrm{N}}=0$ on $\mathcal{F}^{1}$. Differentiating this equation, and an application of the Cartan lemma (see, e.g. [IL03, p. 314]), yields functions $r_{a b}=r_{b a}$ : $\mathcal{F}^{1} \rightarrow \mathbb{C}$ such that

$$
\omega_{a}^{\mathrm{N}}=r_{a b} \omega_{0}^{b}, \quad 1 \leq a, b \leq n .
$$

The coefficients $r_{a b}$ define the Fubini quadric (also known as the projective second fundamental form) $F_{2}=r_{a b} \underline{e}^{a} \underline{e}^{b} \otimes \underline{e}_{\mathrm{N}} \in \Gamma\left(\mathcal{F}^{1}, \pi^{*}\left(S^{2} T^{*} X \otimes N X\right)\right)$. Here the $\underline{e}^{a} \in T_{x}^{*} X$ are dual to the basis

$$
\underline{e}_{a}=e^{0} \otimes\left(e_{a} \bmod e_{0}\right)
$$

of $T_{x} X$ and $x=\pi\left(e_{0}\right)$.

The coefficients of the Fubini cubic are obtained by differentiating (2.1) and another application of Cartan's Lemma. The coefficients $r_{a_{1} a_{2} \cdots a_{p}}$ of the $p$-th Fubini form $F_{p}=$ $r_{a_{1} \cdots a_{p}} \underline{e}^{a_{1}} \cdots \underline{e}^{a_{p}} \otimes \underline{e}_{\mathrm{N}} \in \Gamma\left(\mathcal{F}^{1}, \pi^{*}\left(S^{p} T^{*} X \otimes N X\right)\right)$ are defined inductively. The defining formula is as follows. Let $\mathfrak{S}_{p+q}$ denote the symmetric group on $p+q$ letters. Given two tensors $T_{a_{1} \ldots a_{p}}$ and $U_{a_{p+1} \ldots a_{p+q}}$, let

$$
T_{\left(a_{1} \ldots a_{p}\right.} U_{\left.a_{p+1} \ldots a_{p+q}\right)}=\frac{1}{(p+q) !} \sum_{\sigma \in \mathfrak{S}_{p+q}} T_{\sigma\left(a_{1}\right) \ldots \sigma\left(a_{p}\right)} U_{\sigma\left(a_{p+1}\right) \ldots \sigma\left(a_{p+q}\right)}
$$

denote the symmetrization of their product. For example, $T_{\left(a_{1}\right.} U_{\left.a_{2}\right)}=\frac{1}{2}\left(T_{a_{1}} U_{a_{2}}+T_{a_{2}} U_{a_{1}}\right)$. We exclude from the symmetrization operation any index that is outside the parethenses. For example, in $r_{b\left(a_{1} \ldots a_{p-1}\right.} \omega_{\left.a_{p}\right)}^{b}$ we symmetrize over only the $a_{i}$, excluding the $b$ index. Define

$$
r_{a}=0 \text {. }
$$


Proposition ([Rob08]). The coefficients of $F_{p+1}, p>1$, fully symmetric in their lower indices, are defined by

$$
\begin{aligned}
r_{a_{1} \ldots a_{p} b} \omega_{0}^{b}= & -\mathrm{d} r_{a_{1} \ldots a_{p}}-r_{a_{1} \ldots a_{p}}\left\{(p-1) \omega_{0}^{0}+\omega_{N}^{N}\right\} \\
& +p\left\{(p-2) r_{\left(a_{1} \ldots a_{p-1}\right.} \omega_{\left.a_{p}\right)}^{0}+r_{b\left(a_{1} \ldots a_{p-1}\right.} \omega_{\left.a_{p}\right)}^{b}\right\} \\
& -\sum_{j=1}^{p-2}\left(\begin{array}{c}
p \\
j
\end{array}\right)\left\{r_{b\left(a_{1} \ldots a_{j}\right.} r_{\left.a_{j+1} \ldots a_{p}\right)} \omega_{N}^{b}+(j-1) r_{\left(a_{1} \ldots a_{j}\right.} r_{\left.a_{j+1} \ldots a_{p}\right)} \omega_{N}^{0}\right\} .
\end{aligned}
$$

The Fubini forms $F_{p}$ are defined on $\mathcal{F}^{1}$ and do not descend to well-defined sections of $S^{p} T^{*} X \otimes N X$ over $X$. However, the ideal generated by $\left\{F_{2}, F_{3}, \ldots, F_{k}\right\}$ is independent of our choice of first-order adapted frame over $x \in X$.

Proposition 2.1. Given $X^{n} \subset \mathbb{P}^{n+1}$ and a smooth point $x \in X$, there exists a local framing about $x$ such that all entries of the Maurer-Cartan form (pulled back via this framing) vanish with the exception of $\omega_{0}^{a}$ and $\omega_{a}^{N}, 1 \leq a \leq n$.

Proof. One such local framing is given in $\S 2.4$ below.

Definition 2.2. Given a smooth point $x \in X, \mathcal{C}_{k, x} \subset \mathbb{P} T_{x} X$ is the zero locus of $F_{2, x}, \ldots, F_{k, x}$.

2.4. Fubini forms in a coordinate framing. Given a smooth point $x \in X$, fix homogeneous coordinates $\left[z_{0}: z_{1}: \cdots: z_{\mathrm{N}}\right]$ of $\mathbb{P} V$ so that $x=[1: 0: \cdots: 0]$, and $\widehat{X}$ is tangent to $\left\{z_{N}=0\right\}$ at $(1,0, \ldots, 0) \in \hat{x} \subset V$. Setting $z_{0}=1$ yields a coordinate neighborhood on $\mathbb{P}^{N}$ with local coordinates $\left(z^{1}, \ldots, z^{\mathrm{N}}\right)$ centered at $x$. Shrinking the coordinate neighborhood $U$ if necessary, we may assume that $U \cap X$ is a graph $z^{\mathrm{N}}=f\left(z^{1}, \ldots, z^{n}\right)$ over its embedded tangent space at $x$. A local, first-order adapted frame $e: U \rightarrow \mathcal{F}^{1}$ over $U$ is defined by

$$
\begin{aligned}
e_{0} & =\frac{\partial}{\partial z^{0}}+z^{a} \frac{\partial}{\partial z^{a}}+f \frac{\partial}{\partial z^{N}} \\
e_{a} & =\frac{\partial}{\partial z^{a}}+f_{z^{a}} \frac{\partial}{\partial z^{N}} \\
e_{N} & =\frac{\partial}{\partial z^{N}}
\end{aligned}
$$

here, $f_{z^{a}}$ denotes partial differentiation, and $1 \leq a \leq n$. With respect to this frame, the only nonzero entries in the Maurer-Cartan form $\omega=\omega_{k}^{j} e_{j} \otimes e^{k}$ are

$$
\omega_{0}^{a}=\mathrm{d} z^{a} \quad \text { and } \quad \omega_{a}^{\mathrm{N}}=f_{z^{a} z^{b}} \mathrm{~d} z^{b} .
$$

Regard $z=\left(z^{1}, \ldots, z^{n}\right)$ as local coordinates on $X$. It is immediate from the expression for $\omega_{a}^{\mathrm{N}}$ above that the coefficients of the second Fubini form at $z$ are $r_{a b}(z)=f_{z^{a} z^{b}}(z)$. More generally, Proposition 2.3 implies the Fubini forms at $z$ are given by

$$
F_{k, e}(z)=(-1)^{k} f_{z^{a_{1} \ldots z^{a_{k}}}}(z) \mathrm{d} z^{a_{1}} \cdots \mathrm{d} z^{a_{k}} \otimes \underline{e_{\mathrm{N}}} .
$$

See [IL03, §3.3.7] for details.

If the $y=\left(y^{1}, \ldots, y^{n}\right)$ are linear coordinates on $T_{z} X$ induced by $\left\{e_{1}, \ldots, e_{n}\right\}$, it follows that $\mathcal{C}_{k, z}$ is the zero set of the polynomials

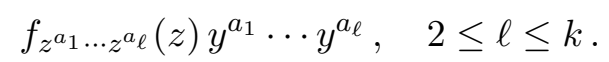


In particular,

$$
\mathcal{C}_{k, x}=\left\{\ell \in \mathbb{P} T_{x} X \mid \exists L=\mathbb{P}^{1} \subset \mathbb{P}^{n}, \mathbb{P} T_{x} L=\ell, \operatorname{mult}_{x}(X \cap L) \geq k+1\right\} .
$$

Lemma 2.3. Given $\mathcal{C} \subset \mathbb{P} T$, a complete intersection of hypersurfaces of degrees $2, \ldots, d$ and $d^{\prime} \geq d$, there exists a hypersurface $X$ of degree $d^{\prime}$, a point $x \in X$, and a local framing e such that $\operatorname{Zeros}\left(\tilde{\phi}_{d, e}(x)\right)=\mathcal{C}$.

Remark. Thus given $\mathbf{P} \in \oplus_{\delta=2}^{d} S^{\delta} T^{*}$ there exist $X$ and $x \in X$ such that $\phi_{d}(x)=\mathbf{P}$. It is not clear which points of $\oplus_{\delta=2}^{d} S^{\delta} T^{*}$ can be the image of a general point of some $X$.

Proof. Pick $\mathbf{P}=\left(P_{2}, \ldots, P_{d}\right)$, with $P_{j} \in S^{j} T^{*}$ so that $\mathcal{C}=[\operatorname{Zeros}(\mathbf{P})]$. Let $f(z)=$ $f\left(z^{1}, \ldots, z^{n}\right)$ be a polynomial of degree $d^{\prime}$ and let $f_{k}(z)$ denote the degree $k$ homogeneous component of $f(z)$. Specify $f_{0}=0, f_{1}=0$ and $f_{\delta}=P_{\delta}$ for $2 \leq \delta \leq d$. Take $X$ to be the closure of the graph of $f$, and $x=[1: 0: \cdots: 0]$.

\section{Results in terms of Fubini forms}

3.1. Gauss maps. We will consider two types of Gauss map: the Gauss map of a hypersurface in $\mathbb{P} V$, and the Gauss map associated to a differentiable map between manifolds. Given a vector space $V$, let $G(m, V)$ denote the Grassmannian of $m$-planes through the origin in $V$.

The Gauss map $\gamma_{X}$ of a hypersurface $X \subset \mathbb{P}^{N}=\mathbb{P} V$ is

$$
\begin{aligned}
\gamma_{X}: X_{\text {smooth }} & \rightarrow G(n+1, V), \\
x & \mapsto \gamma_{X}(x):=\widehat{T}_{x} X .
\end{aligned}
$$

The tangent space $T_{E} G(n+1, V)$ may be identified with $(V / E) \otimes E^{*}$. Making use of a frame $e$ we may identify $T_{\gamma_{X}(x)} G(n+1, V)$ with $N_{x} X \otimes \hat{x} \otimes\left(\widehat{T}_{x} X\right)^{*}$. Under the identification the differential is given by $\mathrm{d} \gamma_{X, x}\left(v^{a} \underline{e}_{a}\right)=v^{a} r_{a b} \underline{e}_{\mathrm{N}} \otimes e^{b} \in N_{x} X \otimes \hat{x} \otimes\left(\widehat{T}_{x} X\right)^{*}$.

The Gauss map $\gamma_{X}$ is nondegenerate if $\mathrm{d} \gamma_{X, x}: T_{x} X \rightarrow T_{\gamma_{X}(x)} G(n+1, V)$ is of maximial rank $n$. The Gauss map is nondegenerate if and only if the quadratic polynomial $F_{2, e}$ is of full rank; equivalently, the quadric hypersurface $\left\{F_{2, e}(x)=0\right\} \subset \mathbb{P} T_{x} X$ is smooth.

Remark. The second Fubini form $F_{2}$ and (projective) second fundamental form (defined by $\left.\mathrm{d} \gamma_{X}\right)$ agree. See [IL03, Ch. 3].

Given a manifold $\Sigma$, let $\mathbf{G}(n, T \Sigma) \rightarrow \Sigma$ denote the Grassmann bundle, whose fiber over $\sigma \in \Sigma$ is $G\left(n, T_{\sigma} \Sigma\right)$. Let $f: Z \rightarrow \Sigma$ be a $C^{1}$ map of manifolds of generic rank $r$ and set $Z^{\prime}=\left\{z \in Z \mid \operatorname{rank}\left(d f_{z}\right)=r\right\} \subset Z$. The Gauss map $\gamma_{f}$ associated to $f$ is

$$
\begin{aligned}
\gamma_{f}: Z^{\prime} & \rightarrow \mathbf{G}(r, T \Sigma) \\
x & \mapsto T_{f(x)} f(Z) .
\end{aligned}
$$

3.2. Results. Given $S=\left(S_{3}, \ldots, S_{d+1}\right) \in \oplus_{\delta=3}^{d+1} S^{\delta} T^{*}$, such that the common zero locus of the $S_{j}$ is not a cone, we obtain an $n$-plane $E_{S} \in G\left(n, \oplus_{\delta=2}^{d} S^{\delta} T^{*}\right)$ by

$$
\left.E_{S}=\left\langle(v\lrcorner S_{3}, \ldots, v\right\lrcorner S_{d+1}\right)|v \in T\rangle .
$$


Here $v\lrcorner: S^{\delta} T^{*} \rightarrow S^{\delta-1} T^{*}$ denotes the interior product, i.e., $\left.(v\lrcorner P\right)\left(w_{1}, \ldots, w_{\delta-1}\right)=P\left(v, w_{1}, \ldots, w_{\delta-1}\right)$. Fix $\mathbf{P}=\left(P_{2}, \ldots, P_{d}\right) \in \oplus_{\delta=2}^{d} S^{\delta} T^{*}$. Define a map

$$
\begin{aligned}
\mu_{\mathbf{P}}: S^{d+1} T^{*} & \rightarrow \oplus_{\delta=3}^{d+1} S^{\delta} T^{*}, \\
\alpha & \mapsto\left(P_{3}, \ldots, P_{d}, \alpha\right) .
\end{aligned}
$$

Let $I_{\delta}(\mathbf{P})$ denote the degree $\delta$ homogeneous component of the ideal generated by $\left(P_{2}, \ldots, P_{d}\right)$.

Definition 3.1. Using the notations $E_{S}, \mu_{\mathbf{P}}$ above, define the varieties

$$
\begin{aligned}
& \boldsymbol{\Xi}_{\mathbf{P}}:=\left\{E_{\mu_{\mathbf{P}}(\alpha)} \mid \alpha \in S^{d+1} T^{*}\right\} \subset G\left(n, \oplus_{\delta=2}^{d} S^{\delta} T^{*}\right) \\
& \mathbf{\Phi}_{\mathbf{P}}:=\left\{E_{\mu_{\mathbf{P}}(\beta)} \mid \beta \in I_{d+1}(\mathbf{P})\right\} \subset G\left(n, \oplus_{\delta=2}^{d} S^{\delta} T^{*}\right) .
\end{aligned}
$$

Since $\oplus_{\delta=2}^{d} S^{\delta} T^{*}$ is a vector space, we may identify the tangent space $T_{\mathbf{P}}\left(\oplus_{\delta=2}^{d} S^{\delta} T^{*}\right)$ with $\oplus_{\delta=2}^{d} S^{\delta} T^{*}$, and define sub-bundles of the Grassmann bundle: $\boldsymbol{\Phi}_{d} \subset \mathbf{\Xi}_{d} \subset \mathbf{G}\left(n, T\left(\oplus_{\delta=2}^{d} S^{\delta} T^{*}\right)\right)$ by $\left(\boldsymbol{\Xi}_{d}\right)_{\mathbf{P}}=\boldsymbol{\Xi}_{\mathbf{P}}$, and $\left(\boldsymbol{\Phi}_{d}\right)_{\mathbf{P}}=\boldsymbol{\Phi}_{\mathbf{P}}$.

Theorem 3.2. Let $X^{n} \subset \mathbb{P}^{n+1}$ be a hypersurface of degree at least d, with nondegenerate Gauss map. Let $U \subset X$ be a d-general open subset admitting a local framing e as in \$2.4, and consider the map

$$
\begin{aligned}
\tilde{\phi}_{d, e}: U & \rightarrow \oplus_{\delta=2}^{d} S^{\delta} T^{*} \\
x & \mapsto\left(F_{2, e}(x), \ldots, F_{d, e}(x)\right) .
\end{aligned}
$$

Assume further that $\gamma_{\tilde{\phi}_{d, e}}$ is defined on $U$; that is, $\operatorname{rank}\left(\left.d \tilde{\phi}_{d, e}\right|_{x}\right)=n$ for all $x \in U$. Then, recalling the varieties $\boldsymbol{\Xi}_{d}$ and $\boldsymbol{\Phi}_{d}$ of Definition 3.1, we have the following:

(a) The Gauss image $\gamma_{\tilde{\phi}_{d, e}}(U)$ is contained in $\boldsymbol{\Xi}_{d}$.

(b) If $\operatorname{deg}(X)=d$, then $\gamma_{\tilde{\phi}_{d, e}}(U) \subset \boldsymbol{\Phi}_{d}$.

Remark. The assumption on the rank of $\mathrm{d} \tilde{\phi}_{d, e}$ is generic; see Proposition 3.3 below.

Proof. Choose a local framing as in Proposition 2.1, then

$$
\begin{aligned}
\mathrm{d} \tilde{\phi}_{d, e}(z) & =\left(\mathrm{d} F_{2, e}(z), \ldots, \mathrm{d} F_{d, e}(z)\right) \\
& =-\left(F_{3, e}(z), \ldots, F_{d+1, e}(z)\right) .
\end{aligned}
$$

Given $z \in U, \gamma_{\tilde{\phi}_{d, e}}(z)$ is the $n$-plane

$$
\left.\left\langle-(v\lrcorner F_{3, e}(z), \ldots, v\right\lrcorner F_{d+1, e}(z)\right)|v \in T\rangle .
$$

This proves Part (a).

If $\operatorname{deg}(X)=d$, then $F_{d+1, e}(x) \in I_{d+1}\left(\mathcal{C}_{d, x}\right)$, establishing Part (b).

Proposition 3.3. Let $X^{n} \subset \mathbb{P}^{n+1}$ be a general hypersurface of degree $d^{\prime} \geq d$ with nondegenerate Gauss map. Let $U \subset X$ be a d-general open subset admitting a local first-order adapted framing e of $X$ as in Proposition 2.1, and consider the map (3.1). Then $\operatorname{rank}\left(\left.\mathrm{d} \tilde{\phi}_{d, e}\right|_{x}\right)=n$ for all $x \in U$.

Proof. By lower semi-continuity it suffices to show that $\operatorname{rank}\left(\left.\mathrm{d} \tilde{\phi}_{d, e}\right|_{x}\right)=n$ for just one $X$. A family of such $X$ is given in $§ 3.3$. 
3.3. Example. Fix linear coordinates $\bar{z}=\left(z^{0}, \ldots, z^{\mathrm{N}}\right) \in \mathbb{C}^{\mathrm{N}+1}$. Set $z=\left(z^{1}, \ldots, z^{n}\right)$ and let $p_{j}(z)$ be a homogeneous polynomial of degree $j=2, \ldots, d$ with $d>2$. Consider the degree $d$ hypersurface $X_{F}$ given by the zero set of:

$$
F(\bar{z})=-\left(z^{0}\right)^{d-1} z^{\mathrm{N}}+\left(z^{0}\right)^{d-2} p_{2}(z)+\left(z^{0}\right)^{d-3} p_{3}(z)+\cdots+p_{d}(z) .
$$

Note that

$$
\left(X_{F}\right)_{\text {sing }}=\left\{z^{0}=0\right\} \cap Z \operatorname{eros}\left(p_{d-1}\right) \cap Z \operatorname{Zeros}\left(p_{d}\right)_{\text {sing }} .
$$

In particular, $X_{F}$ will usually be smooth. The point $x=[1: 0: \cdots: 0] \in \mathbb{P}^{\mathrm{N}}$ lies on $X_{F}$. In the affine coordinate neighborhood $U=\left\{z^{0}=1\right\} \subset \mathbb{P}^{\mathrm{N}}, X$ may be expressed as a graph

$$
z^{\mathrm{N}}=f(z)=p_{2}(z)+\cdots+p_{d}(z) .
$$

Let $e=\left(e_{0}, \ldots, e_{\mathrm{N}}\right)$ be a first-order framing, constructed as in $\S 2.4$.

Let $y=\left(y^{1}, \ldots, y^{n}\right)$ be linear coordinates on $T^{*}$ induced by the frame $e$. Then

$$
F_{\delta, e}(z)=(-1)^{\delta}\left(\frac{\partial^{\delta} f}{\partial z^{a_{1}} \cdots \partial x^{a_{\delta}}}(z)\right) y^{a_{1}} \cdots y^{a_{\delta}} .
$$

If $p_{\delta}(z)=p_{a_{1}, \ldots, a_{\delta}} z^{a_{1}} \cdots z^{a_{\delta}}$, with $p_{a_{1}, \ldots, a_{\delta}}$ symmetric, then the second Fubini quadric is given by $F_{2, e}(z)=r_{a b}(z) y^{a} y^{b}$ with

$$
r_{a b}(z)=2 p_{a b}+\sum_{\delta=3}^{d} j(j-1) p_{a b c_{3} \cdots c_{\delta}} z^{c_{3}} \cdots z^{c_{\delta}} .
$$

It follows that if $p_{2}$ is nondegenerate, then the Fubini quadric will be nondegenerate in a neighborhood of $z=0$.

Additionally, note that $F_{\delta, e}(0)=(-1)^{\delta} \delta ! p_{a_{1} \cdots a_{\delta}} y^{a_{1}} \cdots y^{a_{\delta}}, 2 \leq \delta \leq d$, and $F_{\delta, e}=0$ for all $\delta>d$, so that

$\mathrm{d} \tilde{\phi}_{d, e}(0)(T)=\left\langle\left(3 \cdot 3 ! p_{a b_{1} b_{2}} y^{b_{1}} y^{b_{2}}, \cdots,(-1)^{d-1} d \cdot d ! p_{a b_{1} \cdots b_{d-1}} y^{b_{1}} \cdots y^{b_{d-1}}, 0\right) \mid 1 \leq a \leq n\right\rangle$.

In particular, for a generic choice of $p_{3}$, the differential $\mathrm{d} \tilde{\phi}_{d, e}(0)$ will have maximal rank $n$.

3.4. Exterior differential systems interpretation. We may rephrase Theorem 3.2 in the language of exterior differential systems (EDS) as follows. (See [IL03, p. 177] or $\left[\mathrm{BCG}^{+} 91\right]$.) Let $\Sigma$ be a manifold and let $\pi: \mathbf{G}(n, T \Sigma) \rightarrow \Sigma$ denote the Grassmann bundle. The Grassmannian $\mathbf{G}(n, T \Sigma)$ carries a canonical linear Pfaffian system (J, J). Let $p \in \Sigma$, $E \in \mathbf{G}\left(n, T_{p} \Sigma\right)$, and $E^{\perp} \subset T_{p}^{*} \Sigma$ be the forms vanishing on $E$. The canonical EDS on $\mathbf{G}(n, T \Sigma)$ is generated by the subspace $\mathcal{J} \subset T^{*} \mathbf{G}(n, T \Sigma)$, defined fiber-wise by $\mathcal{J}_{p, E}=$ $\pi^{*}\left(E^{\perp}\right)$. The independence condition $\mathcal{J} \supset \mathcal{J}$ is given by $\mathcal{J}_{p, E}=\pi^{*}\left(T_{p}^{*} \Sigma\right)$. Integral manifolds of this tautological system are immersed $n$-dimensional submanifolds $i: M \rightarrow \mathbf{G}(n, T \Sigma)$ such that $i^{*}(\mathcal{J}) \equiv 0$ and $i^{*}\left(\Lambda^{n}(\mathcal{J} / \mathcal{J})\right)$ is non-vanishing. They are characterized as the Gauss images of immersed $n$-dimensional submanifolds $N$ of $\Sigma$. That is, there exists $N$ such that $M=\gamma(N)$, where $\gamma$ is the Gauss map $p \mapsto\left(p, T_{p} N\right) \in G\left(n, T_{p} \Sigma\right)$. Theorems 3.2.a and 3.2.b are rephrased as

The image $\tilde{\phi}_{d}(X)$ is an integral manifold of the pull-back of the tautological system (J, J) on $\mathbf{G}\left(n, T\left(\oplus_{\delta=2}^{d} S^{\delta} T^{*}\right)\right)$ to $\boldsymbol{\Xi}_{d}$ (respectively, $\mathbf{\Phi}_{d}$ ).

Other results of this paper can similarly be rephrased in the language of EDS. 


\section{Descent to $\mathcal{M}_{d}$}

Fix a polynomial $p$ that occurs as a Hilbert polynomial of a codimension $d-1$ complete intersection of hypersurfaces of degrees $2,3, \ldots, d$ in $\mathbb{P}^{n-1}$ such that the degree two hypersurface is smooth. (Recall that at a general point $x$ on a generic $X, \mathcal{C}_{x}$ with be such a complete intersection.)

Let

and let

$$
\mathcal{U}_{p}=\left\{\left(P_{2}, \ldots, P_{d}\right) \in \oplus_{\delta=2}^{d} S^{\delta} T^{*} \mid \operatorname{Hilb}\left(\operatorname{Zeros}\left(P_{2}, \ldots, P_{d}\right)\right)=p\right\}
$$

$$
\pi_{p}: \mathcal{U}_{p} \rightarrow H_{i l b}
$$

denote the projection map to the Hilbert scheme. Let $X^{n} \subset \mathbb{P}^{n+1}$ be a hypersurface with a nondegenerate Gauss map (§3.1); equivalently the Fubini quadric is of maximal rank at general points. Let $x \in X_{\text {general }}$, and choose a local first-order adapted framing $e$ on an open set $U \subset X$, containing $x$ as in Proposition 2.1. Let $p$ denote the Hilbert polynomial of $\operatorname{Zeros}\left(\tilde{\phi}_{d, e}(x)\right)$. The polynomial $p$ is independent of our choices of $x$ and $e$. Consider the map

$$
\hat{\phi}_{d, e}=\pi_{p} \circ \tilde{\phi}_{d, e}: U \rightarrow \text { Hilb }_{p} .
$$

The map $\hat{\phi}_{d, e}$ depends on our choice of framing. (Recall that our identification of $T_{x} X$ with the fixed vector space $T$ is made via the frame e.) To remove this ambiguity, we would like to quotient the image in $H_{i l b}$ by the action of $G L(T)$. Unfortunately the quotient of $H_{i l b}$ by the action of $G L(T)$ is not a manifold. As mentioned in the introduction, if $X$ is sufficiently general, there is a well-defined quotient of $H i l b_{p}^{0}$. In the case there is a nontrivial stabilizer $G$ of $\hat{\phi}_{d, e}(x) \in H_{i l b}$, let $\mathcal{H}^{G} \subset H_{i l b}$ denote the connected component of Hilb with stabilizer $G$ containing $Z \operatorname{eros}\left(\tilde{\phi}_{d, e}(x)\right)$. In particular $\mathcal{H}^{\mathrm{Id}}=H_{i l b}^{0}$ and when $G=\mathrm{Id}$, we drop the superscript. Note that we are making the implicit assumption that $G$ does not vary with the point $x$.

Set

$$
\mathcal{M}_{d}^{p, G}=\mathcal{H}^{G} / G L(T)
$$

and let

$$
\phi_{d, U}: U \rightarrow \mathcal{M}_{d}^{p, G}
$$

be the quotient map induced by $\hat{\phi}_{d, e}$. This extends to a well defined map

$$
\phi_{d}: X_{\text {general }} \rightarrow \mathcal{M}_{d}^{p, G} \text {. }
$$

Let $\mathcal{S}_{d}^{p, G} \subset \oplus_{\delta=2}^{d} S^{\delta} T^{*}$ denote the inverse image $\hat{\phi}_{d, e}^{-1}\left(\mathcal{H}^{G}\right)$ and let $\boldsymbol{\Xi}_{d}^{p, G}$ and $\boldsymbol{\Phi}_{d}^{p, G}$ denote the images of the bundles $\boldsymbol{\Xi}_{d}$ and $\boldsymbol{\Phi}_{d}$ (Definition 3.1), restricted to $\mathcal{S}_{d}^{p, G}$, and pushed down to $\mathcal{M}_{d}^{p, G}$. Theorem 3.2 implies

Theorem 4.1. Let $X^{n} \subset \mathbb{P}^{n+1}$ be a hypersurface of degree at least d, with nondegenerate Gauss map. Let $x \in X_{\text {general }}$ and let $p$ denote the Hilbert polynomial determined by the Fubini forms of degree $\leq d$. Let $G \subseteq G L(T)$ denote the stabilizer of Zeros $\left(\phi_{d, e}(x)\right)$ in the Hilbert scheme $\mathrm{Hilb}_{p}$, which we assume to be independent of $x \in X_{\text {general }}$, so the map (4.1) is well defined.

(a) Then $\gamma_{\phi_{d}}\left(X_{\text {general }}\right) \subset \boldsymbol{\Xi}_{d}^{p, G}$. 
(b) If $\operatorname{deg}(X)=d$, then $\gamma_{\phi_{d}}\left(X_{\text {general }}\right) \subset \boldsymbol{\Phi}_{d}^{p, G}$.

Theorem 4.2. Let $X^{n} \subset \mathbb{P}^{n+1}$ be either smooth or such that $X$ is $\mathbb{P}^{1}$-uniruled and for a general line $\ell \subset X$, $\ell \cap X_{\text {sing }}=\emptyset$. If for some $d \leq n, \gamma_{\phi_{d}}\left(X_{\text {general }}\right) \subset \boldsymbol{\Phi}_{d}^{p, G}$, then $\operatorname{deg}(X)=d$.

Proof. If $X$ satisfies the hypotheses of the theorem, then $F_{d+1} \subset I\left(\mathcal{C}_{d, x}\right)$ by Definition 3.1. Therefore $\mathcal{C}_{d, x}=\mathcal{C}_{d+1, x}$. By Theorem 2 of [Lan03b], such $X$ are $\mathbb{P}^{1}$-uniruled. But if $\operatorname{deg}(X)>d$, and $B$ is an irreducible variety of lines on $X$ that covers $X$, then every line in $B$ intersects $X_{\text {sing }}$; see, e.g., [LT].

Remark. Compare Theorem 4.2 with Proposition 3.23 of [Lan96]. There, instead of imposing smoothness conditions on $X$, one requires that the generators of $I\left(\mathcal{C}_{d, x}\right)$ have no nontrivial syzygies, and concludes that $X$ has degree $d$ after $2 d+1$ derivatives, as opposed to the $d+1$ derivatives of Theorem 4.2 .

Let $\pi=\pi_{p, G}: \mathcal{S}_{d}^{p, G} \rightarrow \mathcal{M}_{d}^{p, G}$ denote the projection. Set $\mathfrak{m}=\left\{\mathbf{m}=\left(m_{\tau, \delta}\right) \mid m_{\tau, \delta} \in\right.$ $\left.S^{\tau-\delta} T^{*}\right\}$. Then $\mathfrak{m}$ acts on $\oplus_{\delta=2}^{d} S^{\delta} T^{*}$ by

$$
\mathbf{m} .\left(P_{2}, \ldots, P_{d}\right):=\left(P_{2}, P_{3}+m_{3,2} P_{2}, \ldots, P_{d}+\sum_{j=2}^{d-1} m_{d, j} P_{j}\right) .
$$

Additionally, the action of $\mathfrak{g l}(T)$ on $T$ induces an action on $S^{\delta} T^{*}$ and thence on $\oplus_{\delta=2}^{d} S^{\delta} T^{*}$. We denote the action by $X .\left(P_{2}, \ldots, P_{d}\right):=\left(X . P_{2}, \ldots, X . P_{d}\right)$.

Proposition 4.3. With the notation as introduced above, we have

$$
\left.\operatorname{ker} \mathrm{d} \pi\right|_{\mathbf{P}}=(\mathfrak{g l}(T)+\mathfrak{m}) . \mathbf{P}
$$

Proof. The fiber over $\pi(\mathbf{P})$ of the projection $\mathcal{H}^{G} \rightarrow \mathcal{M}_{d}^{p, G}$ is $P G L(T) \cdot I(\mathbf{P})$. The fiber over $\pi_{p}(\mathbf{P})=I(\mathbf{P})$ of the projection from $\mathcal{S}_{d}^{p, G} \rightarrow \mathcal{H}^{G}$ is $M \cdot \mathbf{P}$, where $M$ is the Lie group associated to the Lie algebra $\mathfrak{m}$.

Next, note that given $X \in \mathfrak{g l}(T)$ and $\mathbf{m} \in \mathfrak{m}$ there exists $X^{\prime} \in \mathfrak{g l}(T), \mathbf{m}^{\prime} \in \mathfrak{m}$ such that $X . \mathbf{m} . \mathbf{P}=\mathbf{m}^{\prime} \cdot X^{\prime} . \mathbf{P}$. It now follows that the fiber of the differential $\mathrm{d} \pi$ is the sum of the $\mathfrak{g l}(T)$ and $\mathfrak{m}$ actions.

Proposition 4.4. If $d \geq 4$ and $\mathbf{P} \in \oplus_{\delta=2}^{d} S^{\delta} T^{*}$ is sufficiently general as described in the proof, then for all $E \in \Xi_{\mathbf{P}}$,

$$
E \cap k e r\left(\left.\mathrm{~d} \pi\right|_{\mathbf{P}}\right)=0 .
$$

Proof. Let $\mathbf{P}=\left(P_{2}, \ldots, P_{d}\right)$ be such that $\left(P_{2}, P_{3}, P_{4}\right)$ is a generic triple. Any point of the linear space $E$ is of the form $\left.\left.\left.\left.(v\lrcorner P_{3}, v\right\lrcorner P_{4}, \ldots, v\right\lrcorner P_{d}, v\right\lrcorner \alpha\right)$ for some $v \in T$ and $\alpha \in S^{d+1} T^{*}$. A point of $\operatorname{ker}\left(\left.\mathrm{d} \pi\right|_{\mathbf{P}}\right)$ is of the form

$$
\left(X . P_{2}, X . P_{3}+m_{3,2} P_{2}, \ldots, X . P_{d}+\sum_{j=2}^{d} m_{d, j} P_{j}\right) \text {. }
$$

We can find $X$ such that $\left.X . P_{2}=v\right\lrcorner P_{3}$, but for generic triples $\left(P_{2}, P_{3}, P_{4}\right)$ the vector spaces $\left.\{v\lrcorner P_{4} \mid v \in T\right\}$, which is $n$-dimensional and $X . P_{3}+m_{3,2} P_{2}$ which is less than $\left(n^{2}+n\right)$-dimensional will have zero intersection in the $\left(\begin{array}{c}n+2 \\ 3\end{array}\right)$-dimensional $S^{3} T^{*}$.

Remark. Note that assuming genericity of any of the $P_{\delta}$ for $\delta \geq 4$ would have been enough to conclude (4.2).

Combining Propositions 4.3 and 4.4, we obtain 
Theorem 4.5. For $d \geq 4$, there is a non-empty Zariski open subset $A_{d} \subset \mathcal{M}_{d}$ such that any $X^{n} \subset \mathbb{P}^{n+1}$ of degree at least $d$ with $x \in X_{\text {general }}$ such that $\phi_{d}(x) \in A_{d}$, must have $\operatorname{rank}\left(\left.\mathrm{d} \phi_{d}\right|_{x}\right)=n$.

Remark. More generally, we have

$$
\operatorname{rank} \mathrm{d} \tilde{\phi}_{d, e}(x)-\operatorname{rankd} \phi_{d}(x)=\operatorname{dim}\left(\mathrm{d} \tilde{\phi}_{d, e}(x)(T) \cap(\mathfrak{g l}(T)+\mathfrak{m}) \cdot \tilde{\phi}_{d, e}(x)\right) .
$$

Example 3.3 continued. Generically, the stabilizer of $p_{d}$ in $G L(T)$ will be trivial so that in (4.2) we need only consider the intersection of $\left\langle p_{a b c} y^{b} y^{c}\right\rangle_{a=1}^{n}$ with $\left\langle\left(p_{a b}-p_{a b c} z^{c}\right) y^{a} y^{b}\right\rangle$. Generically, the intersection will be trivial, so that the rank of $\mathrm{d} \phi_{3}$ is maximal.

Remark. Had we normalized $F_{2}$ to be constant, $F_{3}$ would have been forced to vary. See $\S 4.2$.

4.1. Sharper theorems. Instead of descending all the way to $\mathcal{M}_{d}^{p, G}$, one may obtain similar results by descending just to $\left(\oplus_{\delta=2}^{d} S^{\delta} T^{*}\right) / H$ as we have a canonical map $\bar{\phi}_{d}$ : $X_{\text {general }} \rightarrow\left(\oplus_{\delta=2}^{d} S^{\delta} T^{*}\right) / H$. Here $H$ is the unipotent group preserving the flag $\hat{x} \subset \hat{T}_{x} X \subset$ $V$.

4.2. Second order adapted frames. One could choose to work with second order adapted frames where $F_{2, X}$ is normalized to be a fixed quadratic form $Q$ (e.g. $Q=y_{1}{ }^{2}+\cdots+y_{n}{ }^{2}$ ), and consider the map $\tilde{\phi}_{d, e}^{\prime}$ from such a framing to $\oplus_{\delta=3}^{d} S^{\delta} T^{*}$. In this case the derivative $d \tilde{\phi}_{d, e}^{\prime}(z)$ is shifted in the following sense. Contract $P_{\delta} \otimes P_{3}$ via (the dual quadric to) $Q$ to obtain an element of $S^{\delta-1} T^{*} \otimes S^{2} T^{*}$; then symmetrize to get an element $\xi_{\delta}(\mathbf{P}) \in S^{\delta+1} T^{*}$. We find that

$$
\begin{aligned}
\mathrm{d} \tilde{\phi}_{d, e}^{\prime}(z) & =\left(0, \mathrm{~d} F_{3, e}(z), \ldots, \mathrm{d} F_{d, e}(z)\right) \\
& =-\left(F_{3, e}(z)+\xi_{3}(\mathbf{P}), \ldots, F_{d+1, e}(z)+\xi_{d}(\mathbf{P})\right)
\end{aligned}
$$

\section{Cubic examples}

5.1. Fermat cubic. Given linear coordinates $\bar{z}=\left(z^{0}, \ldots, z^{\mathrm{N}}\right) \in \mathbb{C}^{\mathrm{N}+1}$, consider the Fermat cubic

$$
F(\bar{z})=\left(z^{0}\right)^{3}+\cdots+\left(z^{\mathrm{N}}\right)^{3} .
$$

It is easy to see that $x=\left[\mathrm{N}^{1 / 3}:-1: \cdots:-1\right]$ is a smooth point of the hypersurface $X=\{F=0\}$. In the cases $3 \leq n \leq 6$ we used Maple to confirm that the Fubini quadric $F_{2}$ is nondegenerate at $x$, and that $\mathrm{d} \phi_{3}$ has maximal rank at $x$.

5.2. The determinant. In contrast to the hypersurfaces discussed in this article, if $X$ is quasi-homogeneous, i.e., the Zariski closure in $\mathbb{P} V$ of an orbit of a group $G$ acting linearly on $V$, then its differential invariants will be constant on a Zariski open subset. More generally, if it is a $G$-variety for some group $G$, then its differential invariants will be constant along $G$-orbits. For example, consider the the cubic hypersurface $\operatorname{Det}(3) \subset \mathbb{P}^{8}$. Given coordinates

$$
w=\left(\begin{array}{lll}
w^{0} & w^{3} & w^{8} \\
w^{6} & w^{1} & w^{4} \\
w^{5} & w^{7} & w^{2}
\end{array}\right) \in \mathbb{C}^{9},
$$


$\operatorname{Det}(3)$ is given by the equation

$$
F(w)=w^{0} w^{1} w^{2}+w^{3} w^{4} w^{5}+w^{6} w^{7} w^{8}-w^{0} w^{4} w^{7}-w^{2} w^{3} w^{6}-w^{1} w^{5} w^{8} .
$$

We will consider an open coordinate neighborhood of the smooth point $x=[1: 1: 0: \cdots$ : $0] \in \operatorname{Det}(3)$.

Note that Det(3) is preserved by the action of $G L(9)$ on $\mathbb{P}^{8}$ and that $x$, corresponding to a rank two matrix, lies in the maximal orbit. Hence we expect $\phi_{3}$ to be constant. Indeed it is possible to construct a local, first-order adapted framing $e^{\prime}$ in a neighborhood of $x$ with respect to which the Fubini invariants and thus $\tilde{\phi}_{3, e^{\prime}}$ are constant.

However, the framing $e^{\prime}$ is not of the form constructed in §2.4. Given a framing $e$ of the type constructed $\S 2.4, \mathrm{~d} \tilde{\phi}_{3, e}$ will have maximal rank 7 . However, $\mathrm{d} \tilde{\phi}_{3, e}(x)(T) \subset$ $I^{d}(x)+\mathfrak{g l}(T) . \tilde{\phi}_{3, e}(x)$, and (4.3) yields $\operatorname{rank} d \phi_{3, e}(x)=0$.

The first three differential invariants in such a framing are

$$
\begin{aligned}
& F_{2, e}(x)=2\left(y^{3} y^{6}+y^{5} y^{7}\right) \\
& F_{3, e}(x)=6\left(y^{1} y^{3} y^{6}-y^{1} y^{5} y^{7}+y^{2} y^{5} y^{6}+y^{3} y^{4} y^{7}\right), \\
& F_{4, e}(x)=12\left(\left(y^{1}\right)^{2}+y^{2} y^{4}\right) F_{2, e}(z) .
\end{aligned}
$$

Note also that the quadric $F_{2, e}$ is singular. This requires that we alter the moduli space that $\phi_{3}$ maps into.

5.3. The permanent. Maintaining the coordinates of $\S 5.2$, the equation of the permanent $\operatorname{Perm}(3)$ is

$$
F(w)=w^{0} w^{1} w^{2}+w^{3} w^{4} w^{5}+w^{6} w^{7} w^{8}+w^{0} w^{4} w^{7}+w^{2} w^{3} w^{6}+w^{1} w^{5} w^{8} .
$$

While the permanent is not invariant under the action of $G L(9)$ on $\mathbb{P}^{8}$, it is invariant under left and right multiplication by diagonal matrices (with a one dimensional stabilizer) and a permutation group. So we must have rank $\mathrm{d} \phi_{3} \leq 8-(3+3-1)=3$.

If the entries $w^{5}, w^{6}, w^{0}, w^{3}, w^{8}$ in the first column and row of the matrix $w$ are nonzero, then they can be normalized to 1 by the group action. We selected eight normalized points on the permanent, and found that that computations with (4.3) (aided by Maple) yield $\operatorname{rank} \mathrm{d} \phi_{3}(x)=3$ in each case. Thus rank $\mathrm{d} \phi_{3}(x)=3$ at a general point $x$ on the permanent.

\section{REFERENCES}

[AG04] Maks A. Akivis and Vladislav V. Goldberg. Differential geometry of varieties with degenerate Gauss maps. CMS Books in Mathematics/Ouvrages de Mathématiques de la SMC, 18. SpringerVerlag, New York, 2004.

[AG05] Maks A. Akivis and Vladislav V. Goldberg. Dually degenerate varieties and the generalization of a theorem of Griffiths-Harris. Acta Appl. Math., 86(3):249-265, 2005.

$\left[\mathrm{BCG}^{+} 91\right]$ R. L. Bryant, S. S. Chern, R. B. Gardner, H. L. Goldschmidt, and P. A. Griffiths. Exterior differential systems, volume 18 of Mathematical Sciences Research Institute Publications. SpringerVerlag, New York, 1991.

[Car20] Elie Cartan. Sur la déformation projective des surfaces. Ann. Sci. École Norm. Sup. (3), 37:259356, 1920.

[Car92] Élie Cartan. Leçons sur la géométrie projective complexe. La théorie des groupes finis et continus et la géométrie différentielle traitées par la méthode du repère mobile. Leçons sur la théorie des espaces à connexion projective. Les Grands Classiques Gauthier-Villars. [Gauthier-Villars Great Classics]. Éditions Jacques Gabay, Sceaux, 1992. Reprint of the editions of 1931, 1937 and 1937. 
[FP01] Gerd Fischer and Jens Piontkowski. Ruled varieties. Advanced Lectures in Mathematics. Friedr. Vieweg \& Sohn, Braunschweig, 2001. An introduction to algebraic differential geometry.

[Fub16] G. Fubini. Applicabilita proiettiva di due superficie. Rendiconti di Ciroclo matem. .di Palaermo, XLI:135-162, 1916.

[GH79] Phillip Griffiths and Joseph Harris. Algebraic geometry and local differential geometry. Ann. Sci. École Norm. Sup. (4), 12(3):355-452, 1979.

[Hwa01] Jun-Muk Hwang. Geometry of minimal rational curves on Fano manifolds. In School on Vanishing Theorems and Effective Results in Algebraic Geometry (Trieste, 2000), volume 6 of ICTP Lect. Notes, pages 335-393. Abdus Salam Int. Cent. Theoret. Phys., Trieste, 2001.

[IL03] Thomas A. Ivey and J. M. Landsberg. Cartan for beginners: differential geometry via moving frames and exterior differential systems, volume 61 of Graduate Studies in Mathematics. American Mathematical Society, Providence, RI, 2003.

[JM94] Gary R. Jensen and Emilio Musso. Rigidity of hypersurfaces in complex projective space. Ann. Sci. École Norm. Sup. (4), 27(2):227-248, 1994.

[Lan96] J. M. Landsberg. Differential-geometric characterizations of complete intersections. J. Differential Geom., 44(1):32-73, 1996.

[Lan99a] J. M. Landsberg. Algebraic geometry and projective differential geometry, volume 45 of Lecture Notes Series. Seoul National University Research Institute of Mathematics Global Analysis Research Center, Seoul, 1999.

[Lan99b] J. M. Landsberg. Is a linear space contained in a submanifold? On the number of derivatives needed to tell. J. Reine Angew. Math., 508:53-60, 1999.

[Lan99c] J. M. Landsberg. On the infinitesimal rigidity of homogeneous varieties. Compositio Math., 118(2):189-201, 1999.

[Lan03a] J. M. Landsberg. Lines on projective varieties. J. Reine Angew. Math., 562:1-3, 2003.

[Lan03b] J. M. Landsberg. Lines on projective varieties. J. Reine Angew. Math., 562:1-3, 2003.

[LR08] J. M. Landsberg and C. Robles. Fubini's theorem in codimension two. to appear in Crelle., 2008.

[LT] J. M. Landsberg and O. Tommasi. Lines on projective varieties. preprint.

[Rob08] C. Robles. Rigidity of the adjoint variety of $\mathfrak{s l}_{n}$. Differential Geom. Appl., 2008. to appear, math.DG/0608471.

[Sev01] F. Severi. Intorno ai punti doppi impropri di una superficie generale dello spazio a quattro dimensioni, e a suoi punti tripli apparenti. Rend. Circ. Math. Polermo, 15(2):33-51, 1901.

Department of Mathematics, Texas A\&M University, College Station, TX 77843

E-mail address: jml@math.tamu.edu, robles@math.tamu.edu 